EPJ Web of Conferences 97,00011 (2015)

DOI: 10.1051/epjconf/201597000 11

(C) Owned by the authors, published by EDP Sciences, 2015

\title{
Dilepton production from RHIC to the LHC
}

\author{
Torsten Dahms ${ }^{1, a}$ \\ ${ }^{1}$ Excellence Cluster Universe, Technische Universität München, Garching, Germany
}

\begin{abstract}
The goal of ultrarelativistic heavy-ion collisions at RHIC and the LHC is to study the properties of the quark-gluon plasma (QGP), a phase of matter with partonic degrees of freedom. Electromagnetic radiation, in form of photons or lepton pairs, is a penetrating probe that allows the investigation of the full time evolution and dynamics of the produced matter as it does not undergo strong interaction in the final state. The dilepton spectrum is extremely rich in physics sources: Thermal black-body radiation is of particular interest as it carries information about the QGP temperature. Modifications of the spectral functions of light vector mesons are linked to the potential restoration of chiral symmetry in the QGP phase. Correlated lepton pairs from semi-leptonic charm and beauty decays provide additional information about the heavy-quark energy loss. Finally, the suppression of quarkonia in the QGP give access to an independent temperature measurement. In this proceedings, dilepton results from RHIC are reviewed and the status as well as prospects of low-mass dilepton measurements at the LHC are given.
\end{abstract}

\section{Introduction}

Dileptons, i.e. pairs of oppositely charged leptons $\left(e^{+} e^{-}\right.$or $\left.\mu^{+} \mu^{-}\right)$, have proven to be a useful tool to study the properties of the strongly coupled medium created in ultrarelativistic heavy-ion collisions at the Super Proton Synchrotron (SPS), the Relativistic Heavy Ion Collider (RHIC), and most recently the Large Hadron Collider (LHC). Most dileptons result from hadron decays long after the fireball has ceased to exist. However, few of them are produced within the fireball, either in the hadronic phase from the decay of extremely short lived hadrons, such as the $\rho$ meson $(\tau \approx 1.3 \mathrm{fm} / c)$, or as thermal radiation, e.g. in the partonic phase via the annihilation of quark-antiquark pairs. With its short lifetime the $\rho$ spectral function should be very sensitive to the predicted restoration of chiral symmetry inside the fireball. Enhancements of the invariant-mass spectrum of dileptons above the "cocktail" of ordinary hadron decays had been observed in heavy-ion collisions by the CERES and NA60 experiments at the SPS $[1,2]$. The latter was able to precisely determine that the enhancement was consistent with a broadening of the $\rho$ spectral function, but could not be described by a "dropping" mass scenario as originally predicted by Brown and Rho [3].

\section{The picture at RHIC}

At RHIC, the PHENIX experiment presented first measurements of the dielectron continuum in $p p$, $d \mathrm{Au}$, and Au-Au collisions at $\sqrt{s_{\mathrm{NN}}}=200 \mathrm{GeV}$ [4-6]. STAR has measured the dielectron continuum

\footnotetext{
a e-mail: torsten.dahms@cern.ch
} 
in $\mathrm{Au}-\mathrm{Au}$ collisions at five different energies between $\sqrt{s_{\mathrm{NN}}}=19.6$ and $200 \mathrm{GeV}$ [7-10]. Given the higher multiplicities at RHIC, the combinatorial background is much larger than at the SPS, so that the signal-to-background can be as low as $1 \%$ in the mass region of interest. The situation is worsened, in case of PHENIX, by the limited azimuthal acceptance of its two-arm spectrometer, each of which covers only about 90 degrees of the azimuth. Hence, one of the two electrons falls often outside the detector acceptance so that the signal pair is lost while the other electron, if it falls into the acceptance, still contributes to the combinatorial background. The invariant-mass spectrum of background pairs can be estimated with the same-sign pair spectrum that is free of signals from hadron decays ${ }^{1}$. However, the two-arm design of the PHENIX detector leads to different acceptances for opposite-sign and same-sign pairs. A correction for this difference is required before same-sign pairs can be used as background estimate. Assuming that the background is mostly of combinatorial nature, this correction can be calculated by comparing opposite and same-sign spectra obtained from mixed events, in which electrons from one event are combined with electrons from other events with a similar topology (e.g. primary vertex location and collision centrality).
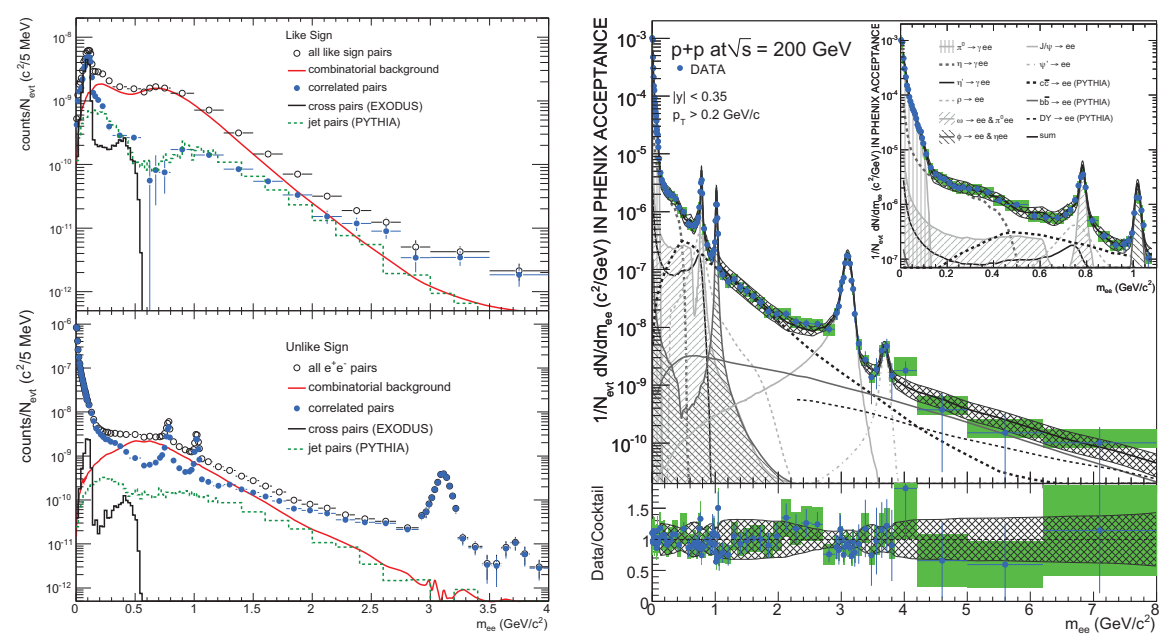

Figure 1. Left: Measured invariant-mass spectrum of $e^{ \pm} e^{ \pm}$(top) and $e^{+} e^{-}$pairs (bottom) in $p p$ collisions at $\sqrt{s}=200 \mathrm{GeV}$. The same-sign pair spectrum is well reproduced by correlated and combinatorial background sources. Right: Invariant-mass spectrum of $e^{+} e^{-}$pairs corrected for reconstruction and trigger efficiencies. The data are well described by a cocktail of expected hadron decays.

Alternatively to the same-sign subtraction, the combinatorial background can be subtracted using the aforementioned mixed event technique. It is important to point out, however, that correlated background sources cannot be reproduced in mixed events. As shown in the left panel of Fig. 1 there are two apparent sources of correlated background, present both in opposite and same-sign spectra. First, so called "cross pairs" from $\pi^{0}$ and $\eta$ Dalitz decays followed by a conversion of the emitted photon in detector material: $\pi^{0} \rightarrow \gamma e^{+} e^{-} \rightarrow e^{+} e^{-} e^{+} e^{-}$. Electrons from the Dalitz decay combined with an electron from the photon conversion will be correlated by the fact that their invariant mass cannot exceed the mass of their parent hadron. The same effect is also present in double Dalitz decays $\left(\pi^{0} \rightarrow e^{+} e^{-} e^{+} e^{-}\right)$though the branching fraction is small compared to the photon conversion

\footnotetext{
${ }^{1}$ At RHIC and even more so at the LHC, $B \bar{B}$ oscillations, followed by semileptonic decays, produce a small signal in the same-sign pair spectrum. Such oversubtraction would need to be considered in the comparison with the hadronic cocktail.
} 
probability in PHENIX. The increasing jet production cross section with centre-of-mass energy adds another correlated background source that has been negligible at the SPS: hadron decays that occur within the same jet or in a back-to-back dijet. The common jet axis introduces a preference for electrons from these hadron decays to have low mass and high $p_{\mathrm{T}}$ (same jet) or high mass and low $p_{\mathrm{T}}$ (dijet). The latter contribution, while present in $p p$ collisions, is strongly suppressed by jet quenching in Au-Au collisions. As these correlated background sources contribute equally to opposite and same-sign spectra, the same-sign subtraction method properly subtracts these sources. Employing a mixed event technique instead makes it necessary to separately calculate spectra of the correlated backgrounds, e.g. based on Monte Carlo simulations that, in $p p$ collisions, adds an uncertainty of $\approx 3 \%$ for $m<1 \mathrm{GeV} / c^{2}$ and $11 \%$ above [4]. The presence of correlated background also complicates the normalisation of the mixed event spectra. The same-sign mixed event spectrum is normalised to the measured same-sign spectrum, in a mass and $p_{\mathrm{T}}$ region free of correlated background (e.g. $m>0.7 \mathrm{GeV} / c^{2}$ for Au-Au collisions). The opposite-sign mixed event spectrum is then scaled to match the geometric mean of the normalised mixed event same-sign yields $\left(2 \sqrt{B_{++} B_{--}}\right)$.

The background subtracted invariant-mass spectrum of $e^{+} e^{-}$in $p p$ collisions at $\sqrt{s}=200 \mathrm{GeV}$ is shown in the right panel of Fig. 1. The data have been corrected for reconstruction and trigger inefficiencies, but without applying a correction for the geometric detector acceptance. The data are compared to a cocktail of expected hadron decays. The cocktail is based on hadron cross sections measured in the same experiment. The $p_{\mathrm{T}}$ differential hadron cross sections are parameterized with a modified Hagedorn function:

$$
E \frac{d^{3} \sigma}{d p^{3}}=A\left(\exp \left(-a p_{\mathrm{T}}-b p_{\mathrm{T}}^{2}\right)+\frac{p_{\mathrm{T}}}{p_{0}}\right)^{-n}
$$

While the combined charged and neutral pion spectra are fitted with all parameters left free, the other hadron spectra $\left(\eta, \omega, \phi, \eta^{\prime}\right.$, and $\left.J / \psi\right)$ can be successfully described with a free normalisation parameter $A$ and $m_{\mathrm{T}}$ scaling, i.e. replacing $p_{\mathrm{T}}$ with the expression $\sqrt{p_{\mathrm{T}}^{2}+m_{h}^{2}-m_{\pi}^{2}}$ where $m_{h}$ and $m_{\pi}$ denote the hadron and pion masses, respectively.

The contributions from semileptonic $D$ and $B$ meson decays as well as Drell-Yan are simulated with the Pyтнia event generator. The charm cross section is set to the cross section measured by PHENIX using non-photonic single electrons: $\sigma_{c \bar{c}}=567 \pm 57$ (stat) \pm 224 (syst) $\mu \mathrm{b}$ [11]. The excellent agreement between data and cocktail allows the subtraction of all but the heavy-flavour contributions from the data. One can then extract the $c \bar{c}$ and $b \bar{b}$ cross sections from a fit to the invariant-mass spectrum [4]:

$$
\begin{aligned}
& \sigma_{c \bar{c}}=518 \pm 47(\text { stat }) \pm 135 \text { (syst) } \pm 200(\text { model }) \mu \mathrm{b} \\
& \sigma_{b \bar{b}}=3.9 \pm 2.4(\text { stat })_{-2}^{+3} \text { (syst) } \mu \mathrm{b} .
\end{aligned}
$$

With the $p p$ baseline understood in terms of hadron decays, the next step is to look for possible modifications in $d$ Au collisions at $\sqrt{s_{\mathrm{NN}}}=200 \mathrm{GeV}$ due to cold nuclear matter effects such as modifications of the nuclear parton distribution functions. No such effects are observed as demonstrated by the good agreement between data and cocktail [6]. One should note that the input cross sections used for the various quarkonium states $(J / \psi, \psi(2 S), \Upsilon(n S))$ are based on measurements in $d$ Au collisions. Hence any modifications of their cross sections by cold nuclear matter effects is already accounted for in the cocktail and not visible in the comparison to data. Utilising additionally the different $p_{\mathrm{T}}$ dependence of $e^{+} e^{-}$pairs from open charm and beauty decays, a $b \bar{b}$ cross section per inelastic nucleon-nucleon collision can be extracted from the data in a 2-dimensional fit to invariant mass and $p_{\mathrm{T}}$ as illustrated in Fig. $2: \sigma_{b \bar{b}}^{N N}=3.4 \pm 0.8$ (stat) \pm 1.1 (syst) \pm 0.11 (model) $\mu$ b. In this 
case not only Pyтнia has been used but also MC@NLO, a next-to-leading order event generator. The difference between the two event generators becomes larger in the measurement of the charm cross section. In contrast to electrons originating from decays of the more massive $b$ quarks, electrons from semileptonic charm decays inherit most of the initial angular correlations from the $c \bar{c}$ pair. Therefore any differences in the $c \bar{c}$ angular correlations introduced by different production mechanisms in the event generator will affect the acceptance correction. Currently, the differences between event generators are too large to perform a measurement of the total charm cross section in $d \mathrm{Au}$.

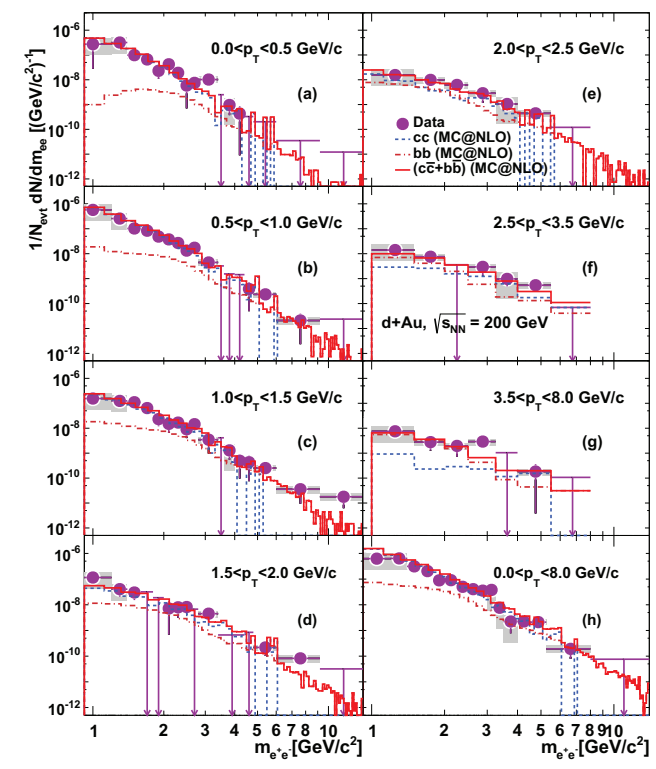

Figure 2. Invariant-mass spectra of $e^{+} e^{-}$pairs from semileptonic heavy flavour decays in bins of $p_{\mathrm{T}}$ measured in $d$ Au collisions at $\sqrt{s_{\mathrm{NN}}}=200 \mathrm{GeV}$. The data are fitted to simulated distributions from MC@ NLO.

The analysis of angular correlations of $e^{ \pm} \mu^{\mp}$ pairs provides another promising approach to measure charm and bottom cross section and to gain a deeper understanding of the production mechanisms. This approach identifies correlated heavy-flavoured mesons that both decayed semileptonically, one into an electron and the other into a muon. By construction, there are no pairs from Dalitz and resonance decays as these are only produced as $e^{+} e^{-}$or $\mu^{+} \mu^{-}$pairs. First results have been presented in $p p$ and $d$ Au collisions by PHENIX [12] that exhibit signs of a modification in $d A u$, possibly due to cold nuclear matter effects such as shadowing in nuclear parton distribution functions.

The dielectron spectrum measured in Au-Au collisions at $\sqrt{s_{\mathrm{NN}}}=200 \mathrm{GeV}$ is shown in Fig. 3 . With the exception of the mass region $150<m<750 \mathrm{MeV} / c^{2}$, the data show an excellent agreement with the hadronic cocktail. The agreement in the open-charm dominated intermediate mass region, between the $\phi$ and the $J / \psi$, with a binary scaled PyтнIA calculation (the same total cross section as for $p p$ case has been used) is a bit surprising, as single electron measurements clearly show that the open charm $p_{\mathrm{T}}$ spectrum is strongly modified in Au-Au collisions [13]. Furthermore, charmed mesons exhibit a strong azimuthal anisotropy. Both effects should lead to a modification (softening) of the invariant mass spectrum. An extreme scenario, in which all $c \bar{c}$ pairs are generated with a random angular correlation and the single electron $p_{\mathrm{T}}$ spectrum matches the measured $\mathrm{Au}-\mathrm{Au}$ spectrum, is 


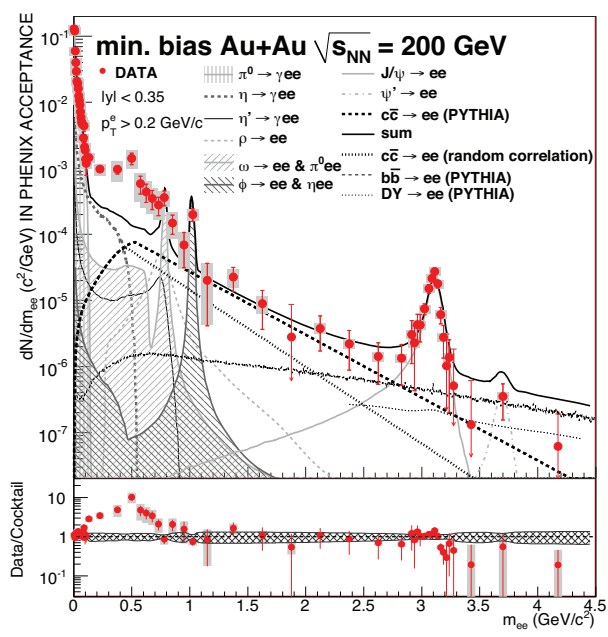

Figure 3. Invariant-mass spectrum of $e^{+} e^{-}$pairs corrected for reconstruction and trigger efficiencies in $\mathrm{Au}-\mathrm{Au}$ (right) collisions at $\sqrt{s_{\mathrm{NN}}}=200 \mathrm{GeV}$. The data exhibit an enhancement above the cocktail in the region $150<$ $m<750 \mathrm{MeV} / c^{2}$.

sketched as dotted line in Fig. 3. It is indeed softer than the default PyтнIA shape and falls below the data, leaving room for a possible thermal contribution from $q \bar{q}$ annihilation.

In the mass region $150<m<750 \mathrm{MeV} / c^{2}$ a strong enhancement by a factor $4.7 \pm 0.4$ (stat) \pm 1.5 (syst) \pm 0.9 (model) above the hadronic cocktail is observed by PHENIX. This enhancement exhibits a pronounced centrality dependence and is concentrated at rather low $p_{\mathrm{T}}$ [5]. Such observation is not corroborated by the STAR experiment [7]. STAR measures a smaller enhancement, shown in the left panel of Fig. 4, that does not depend as strongly on centrality and is well described by theoretical models of a broadened $\rho$ spectral function $[14,15]$. Besides a slightly different mass region, in which the enhancement is defined, one should point out two further differences in the calculation of the "enhancement above hadron cocktail" factor: the charm cross section used by STAR $\left(d \sigma_{c \bar{c}}^{N N} / d y=171 \pm 26 \mu \mathrm{b}\right)$ is a factor 1.4 larger than the one used by PHENIX $\left(d \sigma_{c \bar{c}}^{N N} / d y=123 \pm 12 \pm 37 \mu \mathrm{b}\right)$. With the charm cross section dominating the mass region around $500 \mathrm{MeV} / c^{2}$, the STAR cocktail will account for more of the measured dielectron yield reducing the apparent enhancement. On the other hand, STAR quotes the enhancement above a cocktail that does not include the vacuum $\rho$, while the PHENIX cocktail includes this contribution. It will be interesting to see whether the discrepancy between the low-mass enhancements observed by STAR and PHENIX will be resolved with the data collected by PHENIX with the Hadron Blind Detector upgrade [16].

During the RHIC beam energy scan, STAR has measured the dielectron invariant mass spectrum for five different centre-of-mass energies between $19.6 \mathrm{GeV}$ and $200 \mathrm{GeV}$ [9, 10]. The low-mass dielectron enhancement, present at all collision energies, does not show any dependence with $\sqrt{s_{\mathrm{NN}}}$ as shown in the right panel of Fig. 4. This is expected for $\rho$ modifications that are sensitive to the total baryon density that, in contrast to the net-baryon density, remains relatively constant over the studied energy range. Hence it will be interesting to study dielectron production at even lower centre-of-mass energies, at which the total baryon density will increase. Such programmes are planned for the second beam energy scan RHIC and, with even lower energies, at FAIR [17]. 

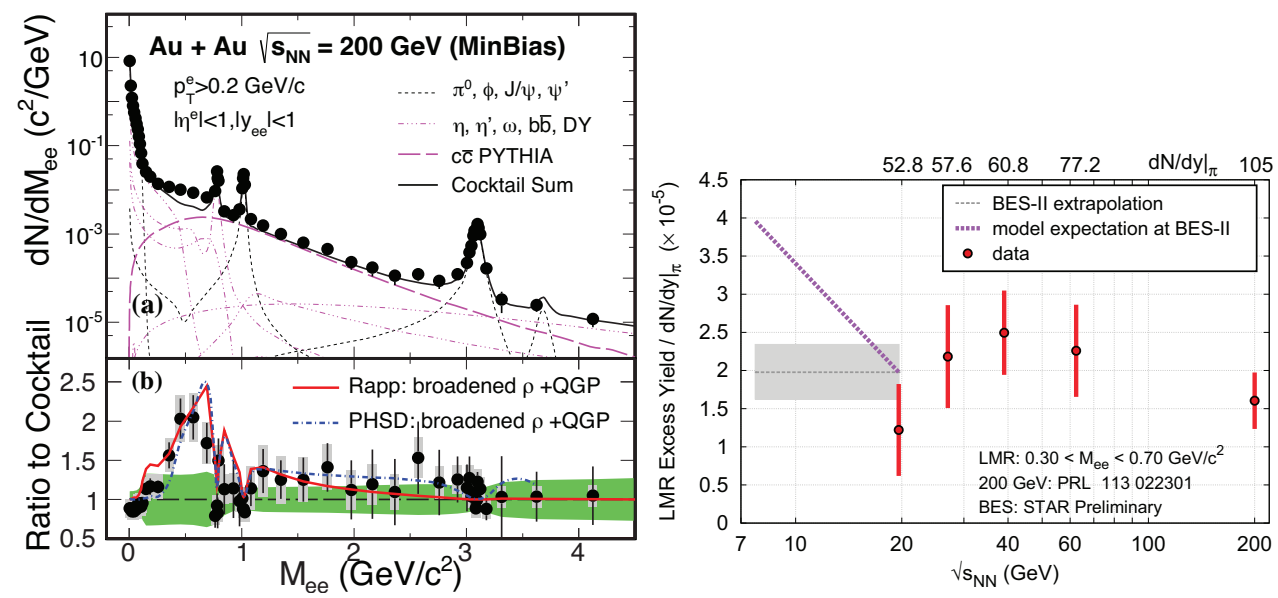

Figure 4. The top left panel shows the invariant-mass spectrum of $e^{+} e^{-}$pairs measured by STAR in Au-Au collisions at $\sqrt{s_{\mathrm{NN}}}=200 \mathrm{GeV}$. The data exhibit an enhancement above the cocktail in the region $300<m<$ $700 \mathrm{MeV} / c^{2}$ quantified as data-to-cocktail ratio in the bottom left panel. The enhancement is well described by theoretical models that include an in-medium broadening of the $\rho$ spectral function. The right panel shows the $\sqrt{s_{\mathrm{NN}}}$ dependence of the enhancement.

STAR has also measured the azimuthal anisotropy of dielectrons in bins of mass and as a function of $p_{\mathrm{T}}$ [8]. For the moment, these measurements do not isolate the enhancement but include dielectrons from hadron decays. The results are in agreement with the known azimuthal anisotropies of hadrons.

Photon conversions in the beam pipe and detector material are a background to dielectron continuum analyses. They can, however, also be used to measure the radiation of thermal photons from a quark-gluon plasma in heavy-ion collisions. PHENIX has published results of an enhanced direct photon signal [18] that nicely complement the analysis of virtual photons [19] and validate the extrapolation from virtual photon to real photon cross sections. The centrality dependence of the direct photon enhancement shows an increase of the yield that scales with the number of participant nucleons as $\approx N_{\text {part }}^{1.5}$. These photons exhibit a strong collective flow [20] that is comparable to hadronic flow. This suggests a rather late emission of these photons, in combination with a significant blue shift of the $p_{\mathrm{T}}$ spectrum, providing hence only information about the temperature close to the phase transition [21]. To gain access to thermal radiation from the earliest and hottest stage, one will need to extract a thermal dilepton signal in the intermediate mass region [22], following the example of NA60 [23].

\section{The future at the LHC}

The energy frontier of heavy-ion collisions is pushed further at the LHC. During the first running period from 2010 to 2013, lead ions were collided at a centre-of-mass energy per nucleon-nucleon pair of $\sqrt{s_{\mathrm{NN}}}=2.76 \mathrm{TeV}$. The upcoming second running period will almost double this energy in the fall of 2015. First attempts to study the dielectron continuum in $p p, p \mathrm{~Pb}$, and $\mathrm{Pb}-\mathrm{Pb}$ collisions have been made. In Fig. 5, dielectron spectra in $p p$ and $p \mathrm{~Pb}$ collisions at $\sqrt{s_{\mathrm{NN}}}=7 \mathrm{TeV}$ and 5.02 TeV, respectively, are compared to hadron cocktails [24]. Both show a good agreement with the expected hadron decay contributions, however, the intermediate mass region shows potential for improving the 
understanding of charm production. The charm contribution to the cocktail is currently limited by the large uncertainties on the total charm cross section measurement.
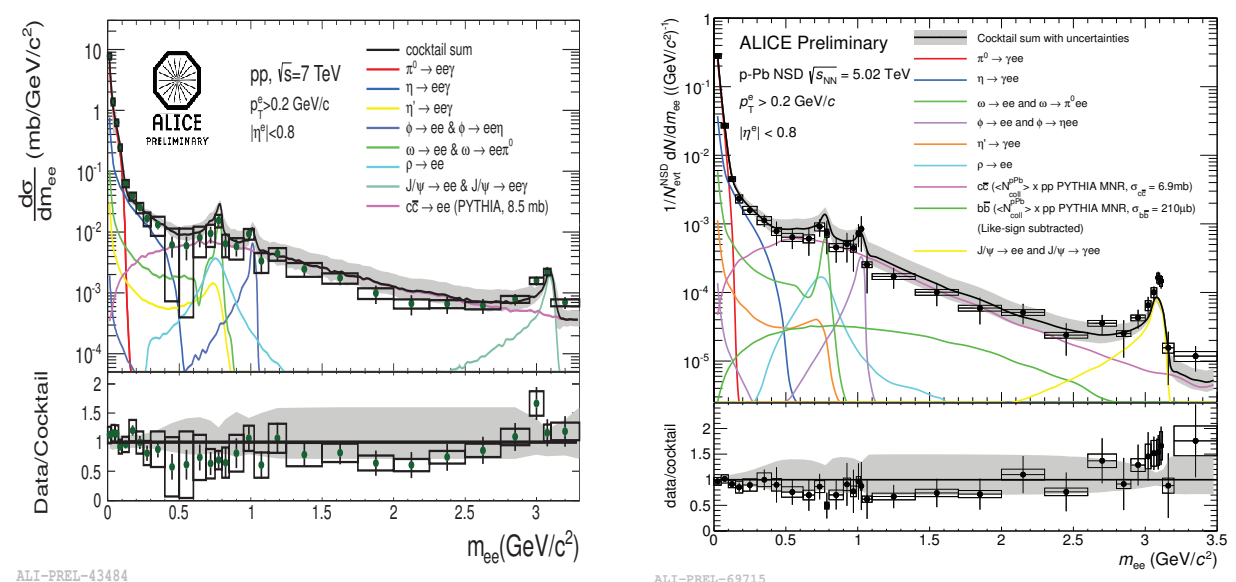

Figure 5. Invariant-mass spectra of $e^{+} e^{-}$pairs measured by ALICE in $p p$ collisions at $\sqrt{s}=7 \mathrm{TeV}$ and $p \mathrm{~Pb}$ collisions at $\sqrt{s_{\mathrm{NN}}}=5.02 \mathrm{TeV}$. The data are compared to a cocktail of expected hadron decays.

The large background rates make the analysis of $\mathrm{Pb}-\mathrm{Pb}$ collisions extremely challenging [24]. Significantly larger data sets than the existing ones are necessary to perform detailed studies of the dielectron continuum in an environment of essentially zero net-baryon density. Such environment provides a unique opportunity to test lattice QCD calculations of chiral symmetry restoration $[25,26]$. Current measurements are limited at low mass to $e^{+} e^{-}$pairs with relatively high $p_{\mathrm{T}}$. Electrons need to have a transverse momentum of at least $0.4 \mathrm{GeV} / c$ in order to reach the time of flight detector, the outermost detector system of ALICE, that plays an important role in the electron identification in $\mathrm{Pb}-\mathrm{Pb}$. This imposes a threshold on the transverse mass of $e^{+} e^{-}$pairs of $m_{\mathrm{T}}=\sqrt{p_{\mathrm{T}}+m}>0.8 \mathrm{GeV} / c$. Plans exist to have a dedicated run with a lowered magnetic field strength that would lower this $m_{\mathrm{T}}$ threshold. Together with the foreseen detector upgrades, most notably the GEM-TPC [27] and the ITS [28], the third running period at the LHC will be most fruitful for the physics of the dielectron continuum. These upgrades will enable ALICE to cope with the foreseen increase in instantaneous luminosity and record $\mathrm{Pb}-\mathrm{Pb}$ collisions at a rate of up to $50 \mathrm{kHz}$. Furthermore, the ITS will be instrumental in identifying non-prompt electrons from semileptonic open heavy flavour decays to separate non-prompt $e^{+} e^{-}$pairs from prompt thermal radiation.

\section{Summary}

Dielectron measurements at RHIC have revealed a number of highly interesting results. The collisionenergy (in)dependence of the low-mass enhancement between $\sqrt{s_{\mathrm{NN}}} \approx 20$ and $200 \mathrm{GeV}$ further supports that the source is a modification of the $\rho$ spectral function. Signs of thermal radiation provide evidence of a thermalised system above the phase transition temperature, despite the complications caused by the collective flow effects. Future measurements at the LHC will provide an excellent test bench for models so far successful in describing data at the SPS and RHIC. 


\section{Acknowledgements}

The author was supported by the DFG cluster of excellence "Origin and Structure of the Universe".

\section{References}

[1] G. Agakichiev et al. (CERES Collaboration), Eur. Phys. J. C 41, 475 (2005), nucl-ex/0506002

[2] R. Arnaldi et al. (NA60 Collaboration), Eur. Phys. J. C 61, 711 (2009), 0812 . 3053

[3] G. Brown, M. Rho, Phys. Rev. Lett. 66, 2720 (1991)

[4] A. Adare et al. (PHENIX Collaboration), Phys. Lett. B 670, 313 (2009), 0802 . 0050

[5] A. Adare et al. (PHENIX Collaboration), Phys. Rev. C 81, 034911 (2010), 0912 . 0244

[6] A. Adare et al. (PHENIX Collaboration), Phys. Rev. C 91, 014907 (2015), 1405.4004

[7] L. Adamczyk et al. (STAR Collaboration), Phys. Rev. Lett. 113, 022301 (2014), 1312 . 7397

[8] L. Adamczyk et al. (STAR Collaboration), Phys. Rev. C 90, 064904 (2014), 1402 . 1791

[9] P. Huck (on behalf of the STAR Collaboration), Nucl. Phys. A 931, 659 (2014), 1409. 5675

[10] L. Adamczyk et al. (STAR Collaboration) (2015), 1501.05341

[11] A. Adare et al. (PHENIX Collaboration), Phys. Rev. Lett. 97, 252002 (2006), hep-ex/0609010

[12] A. Adare et al. (PHENIX Collaboration), Phys. Rev. C 89, 034915 (2014), 1311. 1427

[13] A. Adare et al. (PHENIX Collaboration), Phys. Rev. C 84, 044905 (2011), 1005.1627

[14] R. Rapp, PoS CPOD2013, 008 (2013), 1306. 6394

[15] O. Linnyk, W. Cassing, J. Manninen, E. Bratkovskaya, C. Ko, Phys. Rev. C 85, 024910 (2012), 1111.2975

[16] E. T. Atomssa (on behalf of the PHENIX Collaboration), Nucl. Phys. A 904-905, 561c (2013)

[17] C. Höhne (on behalf of the CBM Collaboration), Nucl. Phys. A 931, 735 (2014)

[18] A. Adare et al. (PHENIX Collaboration) (2014), Submitted to Phys. Rev. C., 1405 . 3940

[19] A. Adare et al. (PHENIX Collaboration), Phys. Rev. Lett. 104, 132301 (2010), 0804.4168

[20] A. Adare et al. (PHENIX Collaboration), Phys. Rev. Lett. 109, 122302 (2012), 1105.4126

[21] C. Shen, U.W. Heinz, J.F. Paquet, C. Gale, Phys. Rev. C 89, 044910 (2014), 1308 . 2440

[22] R. Rapp, Acta Phys. Polon. B 42, 2823 (2011), 1110. 4345

[23] R. Arnaldi et al. (NA60 Collaboration), Phys. Rev. Lett. 100, 022302 (2008), 0711. 1816

[24] M. K. Köhler (on behalf of the ALICE Collaboration), Nucl. Phys. A 931, 665 (2014)

[25] G. Aarts, C. Allton, J. Foley, S. Hands, S. Kim, Phys. Rev. Lett. 99, 022002 (2007), hep-lat/0703008

[26] H.T. Ding, A. Francis, O. Kaczmarek, F. Karsch, E. Laermann et al., Phys. Rev. D 83, 034504 (2011), 1012. 4963

[27] B. Abelev et al. (ALICE Collaboration), Tech. Rep. CERN-LHCC-2013-020. ALICE-TDR-016, CERN, Geneva (2013)

[28] B. Abelev et al. (ALICE Collaboration), Tech. Rep. CERN-LHCC-2013-024. ALICE-TDR-017, CERN, Geneva (2013) 